\title{
Demonstration of NFV for Mobile Edge Computing on an Optically Disaggregated Datacentre in a Box
}

\author{
Michael Enrico' ${ }^{1}$, Vaibhawa Mishra ${ }^{2}$, Arsalan Saljoghei ${ }^{2}$, Maciej Bielski ${ }^{3}$, Evert Pap ${ }^{4}$, Ilias Syrigos ${ }^{5}$, Oscar \\ González de Dios ${ }^{6}$, Dimitris Theodoropoulos ${ }^{7}$, Dionisios N, Pnevmatikatos ${ }^{7}$, Andrea Reale ${ }^{8}$, Dimitris \\ Syrivelis $^{8}$, Georgios Zervas ${ }^{2}$, Nick Parsons ${ }^{1}$, Kostas Katrinis ${ }^{8}$ \\ ${ }^{1}$ HUBER+SUHNER Polatis Ltd, ${ }^{2}$ University College London, ${ }^{3}$ Virtual Open Systems SAS, ${ }^{4}$ Sintecs BV, ${ }^{5}$ University of Thessaly, \\ ${ }^{6}$ Telefonica Investigación y Desarrollo SA, ${ }^{7}$ Foundation for Research and Technology - Hellas, ${ }^{8}$ IBM Research - Ireland \\ michael.enrico@polatis.com
}

\begin{abstract}
This demonstrator showcases the hardware and software integration achieved by the dReDBox project [1] towards realization of a novel architecture using dynamically-reconfigurable optical interconnects to create a flexible, scalable and efficient disaggregated datacentre infrastructure.
\end{abstract}

\section{Overview}

The dReDBox project aims to change the way in which datacentres are built, proposing the deployment of pooled, disaggregated resources rather than monolithic and tightly integrated server-centric components. The overall goal is to improve utilization, scalability, reliability in cloud and edge datacenters to realise cost and energy efficiency benefits. It does this through design and development of a vertical approach for a flexible modular "datacentre-in-abox" architecture. This starts from the hardware platform, including the FPGA firmware necessary to realize the disaggregation of CPU and other functions (memory and acceleration), together with dynamic optically-switched connectivity and mid-board silicon photonics (SiP) transceiver optics. The development further encompasses enhancements to driver software at the host and guest operating system kernel level and finally includes development of applications at the platform management layer as well as enhancements to existing cloud orchestration platforms such as OpenStack.
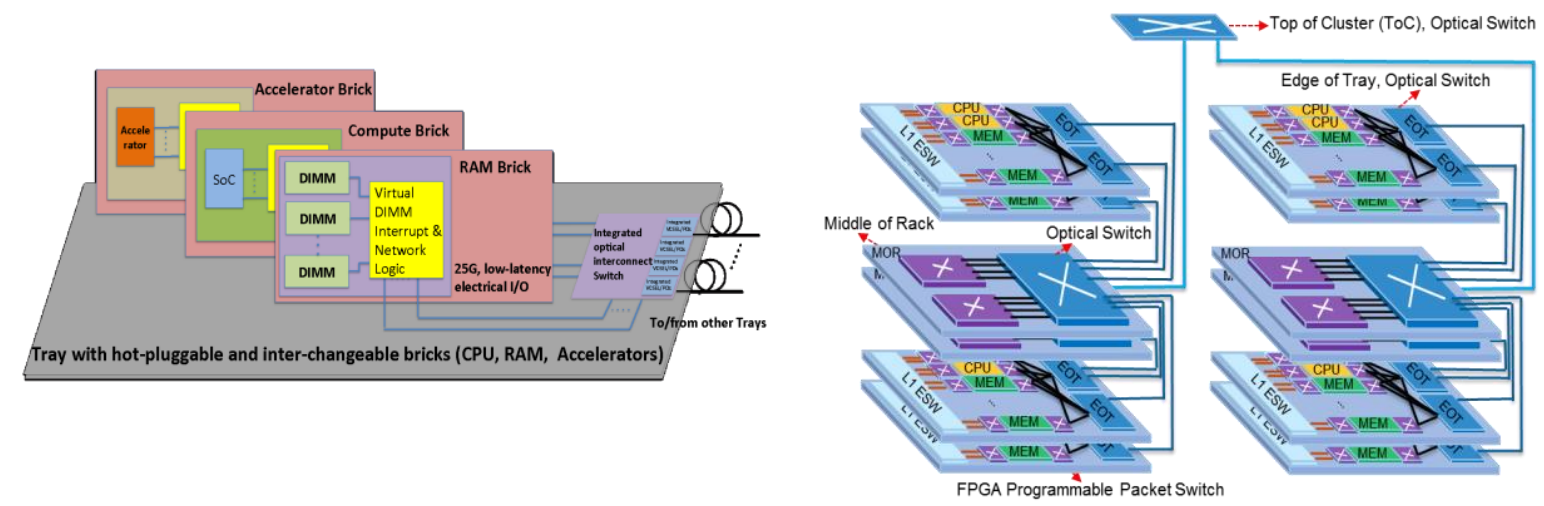

Figure 1: (a) dReDBox bricks (dBRICKs) fitted in a dTRAY, and (b) two dReDBox racks (dRACKs) showing two stages of optical circuit switching.

The modularity is realized as stackable $2 \mathrm{U}$ high "boxes" (dBOXes) in which trays (motherboards) can accommodate arbitrary combinations of up to 16 hot-pluggable and interchangeable "bricks" each optimized for compute, memory or acceleration. This is shown in Figure 1(a). The dBRICKs rely on low latency interconnect through a reconfigurable, software-controlled switching infrastructure. This takes the form of an electrical cross-bar for interconnection within the $\mathrm{dBOX}$ and, for interconnect at the rack or datacentre level, a compact, low power, low loss, multistage optical fibre circuit switching fabric to interconnect high speed SiP based optical transceivers integrated onto each of the bricks.

Three applications have been selected in the project to demonstrate the advantages of the dReDBox architecture: (i) video surveillance analytics hosted in the cloud, (ii) secure key management for NFV in the context of mobile edge computing and (iii) real time and off-line network traffic analytics at rates up to 100Gbps. 


\section{Innovation}

The various innovations in the project being made at all the layers of the vertically integrated stack are illustrated in Figure 2. As well as the aforementioned server hardware and software enhancements there are further innovations being made in the optical interconnect. A compact and energy-efficient 96-port low loss optical circuit switch module is being developed for the first stage of switching integrated into the dBOX. Tuning of the SiP optical transceivers and studies of the effects of using different possible resource allocation algorithms that are used as part of the SDM orchestrator along with studies of different multistage optical switching platform designs have together shown that it is easily possible to scale dReDBox installations up to millions of bricks using the generic topology of Figure 1(b). Early results from this work have previously been reported at OFC [2].

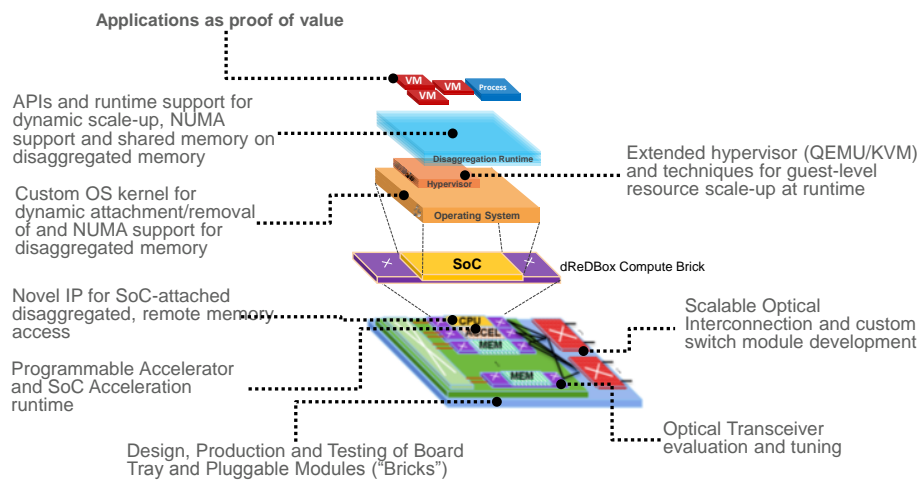

Figure 2: Stack view of key innovations in dReDBox

\section{Demonstration}

The fully-integrated dReDBox hardware is currently under development. The demonstration will therefore be largely based on two FPGA MPSoC evaluation boards representing a compute brick and a remote memory brick. The optical interconnection between these two bricks will use a pair of SiP-based mid-board optics (representing the transceivers that will be integrated onto the bricks), a pair of optical switching modules (representing the $1^{\text {st }}$ stage switching modules that will be integrated into the $\mathrm{dBOX}$ ) and an $\mathrm{NxN}$ optical switch representing a $2^{\text {nd }}$ stage midrack optical switch. These will all be controlled by a Software Defined Memory controller [3] running at the platform management layer which will be used to perform initial VM creation and subsequent runtime VM resizing, demonstrating the hot-plugging of remote network-attached memory. This control will be mediated via an integrated board management controller (dBMC) already developed within the project.

\subsection{Use case}

The application that will be shown on the demonstration hardware will focus on virtualized network functions (VNFs) in the context of mobile edge computing and how they can benefit from the enhanced elasticity inherent in a dReDBox datacentre - in particular, how the databases holding certificate-keys, content and DNS can leverage remote memory to scale up to millions of entries and requests per second, instead of having to scale out and having to replicate very sensitive data.

\section{Relevance to OFC}

The OFC community will be interested in the work of dReDBox because of the use of open software projects and leading edge optical integration. The optical interconnect network is controlled using SDN technology and VMs are provisioned and controlled using an extended OpenStack environment. The demonstrator will show how optics will be essential to realize tangible OPEX and capex reduction in hyperscale and mobile edge datacentre architectures.

\section{References}

[1] K. Katrinis, et al. "Rack-scale disaggregated cloud data centers: The dReDBox project vision." 2016 Design, Automation \& Test in Europe Conference \& Exhibition (DATE). IEEE, 2016.

[2] G. Zervas, et al. "Disaggregated Compute, Memory and Network Systems: A New Era for Optical Data Centre Architectures", OFC 2017 (Invited).

[3] D. Syrivelis, et al. "A Software-defined Architecture and Prototype for Disaggregated Memory Rack Scale Systems ", International Conference on Embedded Computer Systems: Architectures, Modeling, and Simulation, 2017. 